\title{
Acceleration and Dissipation in Relativistic Winds
}

\author{
Jonathan Arons \\ University of California, Berkeley, Department of Astronomy, 601 \\ Campbell Hall, Berkeley, CA 94720-3411, USA
}

\begin{abstract}
I argue that ideal MHD relativistic winds are always limited in practice to asymptotic 4-velocity $\gamma_{\infty} \approx \sigma_{0}^{1 / 3}$ and asymptotic magnetization $\sigma \sim \sigma_{0}^{2 / 3} \gg 1$, where $\sigma_{0}$ is the wind magnetization with respect to the rest energy density, evaluated at the light cylinder of the rotating, magnetized compact object that drives the flow. This suggests that the observed low value of the asymptotic $\sigma$ in the equatorial sectors of the winds driving pulsar wind nebulae and the associated high values of the asymptotic 4-velocity are a consequence of magnetic dissipation in the wind zone.
\end{abstract}

\section{Introduction}

Pulsar wind nebulae (PWNe) provide our nearest at hand examples of relativistic outflows from compact objects. They also form incontrovertible examples of electromagnetically driven flows. Thus their physics is of interest, both in its own right and as purveyors of insight into more remote systems known to have relativistic outflow dynamics, such as AGN and GRB jets, and suspected of being electromagnetically driven.

\section{Observations of PWNe Relevant to Relativistic Wind Physics}

Observations and models of young PWNe allow us to diagnose the properties of the relativistic winds conveying the underlying compact objects' rotational energy to their nebulae. 1D flow models applied to the Crab Nebula 20 years ago by Kennel \& Coroniti (1984a, b) led to the conclusion that the equatorial relativistic wind from the Crab pulsar just upstream of the wind's termination shock has some surprising properties: a terminal Lorentz factor $\gamma_{1} \approx 10^{6}$ and a terminal magnetization $\sigma_{1}=B_{1}^{2} / 4 \pi \gamma_{1} \rho_{1} c^{2} \approx 3 \times 10^{-3} \ll 1$. Such models also lead to a measurement of the $e^{ \pm}$injection rate $\left(\dot{N}_{ \pm} \sim 10^{38.5} \mathrm{~s}^{-1}\right.$ for the $\mathrm{Crab})$ which is in reasonable accord with previous inferences from applications of elementary synchrotron theory to nebular X-ray emission. Similar inferences have been drawn for other PWNe (Gaensler et al. 2002; but see Reynolds 2003). Modeling (Spitkovsky \& Arons 2004) of the time dependent structure observed in the shock termination layer (Hester et al. 2002) comes up with very similar values for $\gamma_{1}, \sigma_{1}$ and $\dot{N}_{ \pm}$. Such models make the additional suggestion that the equatorial wind has a high energy ion component with energy per particle 
$E_{i} \approx q_{i} \Phi / m_{e f f} c^{2}$, where $\Phi$ is the rotational voltage of the pulsar. The inferred ion injection rate $\dot{N}_{i}$ is about equal to the electric current per unit charge expected from electrodynamic theories of magnetized compact object spindown (the Goldreich-Julian current). Here $m_{\text {eff }}=m_{i}+2 \kappa_{ \pm} m_{ \pm}$is the rest mass per particle of the plasma in the relativistic wind, with $\kappa_{ \pm}$the number of pairs per Goldreich-Julian current carrier expelled from the underlying star. Under pulsar conditions, $m_{i}=m_{p}$ or $m_{H e}$ and $m_{e f f}=(5-10) m_{p}$. Such models fail to account for the higher average particle flux required to explain the Crab Nebula's radio emission; see Arons (1998) and Gallant et al. (2002) for discussions of this issue.

\section{Wind Acceleration Theory}

The high wind 4-velocity $\gamma_{1}$ and the low magnetization $\sigma_{1}$ generate problems for the basic physics of relativistic MHD wind flow; the $\dot{N}_{ \pm}$problem may point to troubles with the theory of the plasma source. The existing theory of MHD wind flow (Michel 1969; Goldreich \& Julian 1970; Beskin, Kuznetsova \& Rafikov 1998), applied to rotators with split monopole poloidal magnetic field geometry, suggests that the wind 4-velocity at "infinity" is $\gamma_{\infty} \approx \sigma_{0}^{1 / 3}$, where $\sigma_{0} \approx B_{L}^{2} / 4 \pi \rho_{L} c^{2} \approx e \Phi / 2 m_{e f f} c^{2}$. Contopoulos, Kazanas \& Fendt (1999) show that at least for the aligned rotator, the asymptotic wind structure $(r>$ a few $R_{L}=c / \Omega$ ) is reasonably well approximated by outflow in (split) monopole magnetic geometry, which is almost radial, and Bogovalov's (1999) generalization of split monopole outflow to oblique rotator geometry suggests that almost radial outflow has general applicability. Ideal MHD wind theory applied to more general asymptotic flow structures also suggests almost radial flow outside the light cylinder (Begelman \& Li 1994; Beskin et al. 1998), in which case $\sigma_{\text {wind }}=\sigma_{0} / \gamma_{\text {wind }} \gg 1$ so long as $\gamma_{\text {wind }} \ll \sigma_{0}$. The traditional MHD solutions in monopole geometry with $\gamma_{\text {wind }}(r)<\sigma_{0}^{1 / 3}$ thus imply $\sigma_{\text {wind }}(r)>\sigma_{0}^{2 / 3}$. Everything that has appeared on pulsar magnetosphere theory suggests $\sigma_{0} \gg 1-$ for the Crab, pair creation models (either polar cap or outer gap) suggest $\sigma_{0} \sim 10^{4}$. Thus standard theory says the wind should be "high sigma", all the way to the termination shock, in flagrant contradiction of the inferred magnetization.

The origin of this theoretical prediction is interesting. Magnetic pressure, in the form of a wound up magnetic "spring", provides the driving force underlying outflows driven by large scale electromagnetic fields. The magnetic spring can be an effective accelerator only so long as the magnetic field remains dynamically coupled to the underlying rotator - by Newton's third law, the magnetic field has to push in against a heavy weight (the compact object), in order to exert an outward push on the wind's plasma. Such coupling requires the field at large radii to be able to communicate with the field's roots in the star, which means that acceleration persists only so long as the plasma velocity is less than the magnetosonic speed.

Non-relativistically, such good contact translates into the requirement $\rho v^{2}<$ $B^{2} / 4 \pi$, where $\rho$ is the rest mass density, or $v<v_{m s}=B / \sqrt{4 \pi \rho}=$ nonrelativistic magnetosonic speed. Naively, one might expect that the relativistic version of this constraint would be $\rho \gamma_{\text {wind }} c^{2}<B^{2} / 4 \pi$, or $v_{m s} \rightarrow B / \sqrt{4 \pi \gamma_{\text {wind }} \rho}$ in the 
relativistic case. With $B \propto 1 / r$ and $\rho \propto 1 / r^{2}$, one would then get acceleration until $\gamma_{\text {wind }} \approx \sigma_{0}$ and $\sigma(r) \rightarrow 1$.

This expectation is not correct. Acceleration continues until the wind speed reaches the magnetosonic speed. The acceleration is parallel to the poloidal field and the poloidal velocity. The rate of change of the poloidal speed is expressed by

$$
\rho \frac{d\left(\gamma v_{p}\right)}{d t}=\rho c^{2} \beta_{p} \frac{\partial}{\partial r} \frac{\beta_{p}}{\sqrt{1-\beta_{p}^{2}}}=\rho c^{2} \gamma^{3} \beta_{p} \frac{\partial \beta_{p}}{\partial r} \sim \frac{\rho c^{2} \gamma^{3} \beta_{p}^{2}}{r}
$$

- that is, the accelerating magnetic force must overcome the longitudinal inertial mass/volume $\rho \gamma^{3}$ of the plasma, since the force is parallel to the outflow velocity. Therefore, acceleration ceases once $\gamma$ rises to $\left(B^{2} / 4 \pi \rho c^{2}\right)^{1 / 3}=\sigma_{0}^{1 / 3}$, not to $\sigma_{0}$. This magnetosonic speed based on the longitudinal mass $v_{m s}=B / \sqrt{4 \pi \rho \gamma^{3}}$ has appeared in all the formal discussions of relativistic wind outflow, always without physical explanation. Since this physics is generic to all outflow geometries, all ideal MHD relativistic winds should have asymptotic 4-velocity limited to $^{1} \gamma_{\infty} \sim \sigma_{0}^{1 / 3}$.

The location of the magnetosonic radius, where $v\left(R_{m s}\right)=v_{m s}\left(R_{m s}\right)$, is sensitive to magnetic geometry. In the 1D flow models (Michel 1969; Goldreich \& Julian 1970), $R_{m s}=\infty$ in cold flows. In multi-dimensional models, the inner regions of the flow are force free, with $\gamma \approx r / R_{L}$, (Buckley 1977; Beskin et al. 1998; Contopoulos \& Kazanas 2002; Arons, in preparation). Such "surf-riding", with particles stuck to accelerating field lines, persists until $v=v_{m s}$, which, in monopole geometry, occurs at $R_{m s} \approx \sigma_{0}^{1 / 3} R_{L}$; at larger radii, the wind coasts, with $^{2} \gamma \approx \sigma_{0}^{1 / 3}$. One can readily show that in magnetic geometries with poloidal field lines flaring more rapidly than those of a monopole even for a limited range of radii beyond the light cylinder $R_{m s}$ moves in to be close to the light cylinder (Arons, in preparation).

Thus ideal MHD wind theory leaves both high asymptotic $\gamma$ and low asymptotic $\sigma$ quite unexplained, and will always be an inadequate theory to understand the most elementary interpretation of the observations.

\section{Dissipation}

Arguments such as these suggest that dissipation of magnetic energy in the asymptotic wind zone $R_{L} \ll r<R_{\text {shock }}$ must occur, in order to understand the observed high $\gamma_{1}$ and low $\sigma_{1}$.

The restriction of the observed low $\sigma$, high $\gamma$ flow to the rotational equator is an important clue. The equatorial zone is the likely location of a current sheet separating opposite magnetic polarities in opposite hemispheres. Current sheets

\footnotetext{
${ }^{1}$ In magnetic geometries with field lines focused toward the magnetic axis, weak logarithmic acceleration can occur beyond the radius where the velocity reaches $v_{m s}$ (Begelman \& $\mathrm{Li}$ 1994), but this effect of hoop stress does not have much practical significance.

${ }^{2}$ Contopoulos \& Kazanas (2002) and Arons (2003, 2004) suggested that acceleration in the force free fields continues until equipartition, $\gamma \rightarrow \sigma_{0}$. This is wrong.
} 
are almost always sites of magnetic dissipation. In oblique rotators, this sheet should be wrinkled, taking the form of a frozen-in wave in a sector around the rotational equator (Bogovalov 1999). Possible dissipation mechanisms include some form of reconnection (Kirk \& Skjæraasen 2003), dissipation of the wrinkles at the termination shock (Lyubarsky 2003), if they survive all the way to the shock, and instability of the current sheet with respect to the emission of strong electromagnetic waves which propagate with respect to the wind (Arons 2003,2004 ), which rapidly dissipate (Melatos 1998). While all such mechanisms suggest substantial photon emission, relativistic beaming means that unless the observer lies within the restricted rotational latitude range filled by the dissipative sheet $\left(|\lambda|<10^{\circ}\right.$ in the Crab), the photon signature of the dissipation would be missed. Finding a high voltage pulsar observed from close to the equatorial plane would greatly improve our ability to model these phenomena.

Acknowledgments. I acknowledge assistance from NASA grants NAG512031 and HST-AR-09548.01-A, the Miller Institute for Basic Research in Science and the taxpayers of California.

\section{References}

Arons, J. 1998, Mem. Soc. Ast. It., 69, 989

- 2003, ApJ, 589, 871

- 2004, Adv. Sp. Res., 33, 466

Begelman, M. C., \& Li, Z.-Y. 1994, ApJ, 426, 269

Beskin, V. S., Kuznetsova, I. V., \& Rafikov, R. R. 1998, MNRAS, 299, 341

Bogovalov, S. V. 1999, A\&A, 349, 1017

Buckley, R. 1977, Nature, 266, 37

Contopoulos, I., Kazanas, D., \& Fendt, C. 1999, ApJ, 511, 351

Contopoulos, I., \& Kazanas, D. 2002, ApJ, 566, 336

Gaensler, B. M., Arons, J., Kaspi, V. M., Pivovaroff, M. J., Kawai, N., \& Tamura, K. 2002, ApJ, 569, 878

Gallant, Y. A., van der Swaluw, E., Kirk, J. G., \& Achterberg, A. 2002, in ASP Conf. Ser., Vol. 271, Neutron Stars and Supernova Remnants, eds. P. O. Slane, \& B. M. Gaensler, (San Francisco: ASP), p. 99

Goldreich, P., \& Julian, W. H. 1970, ApJ, 160, 971

Hester, J. J., et al. 2002, ApJ, 577, L49

Kennel, C. F., \& Coroniti, F.C. 1984a, 283, 694

$-1984 \mathrm{~b}, 283,710$

Kirk, J. G., \& Skjæraasen, O. 2003, ApJ, 591, 366

Lyubarsky, Y. E. 2003, MNRAS, 345, 153

Melatos, A. 1998, Mem. Soc. Ast. It., 69, 1009

Michel, F. C. 1969 , ApJ, 158, 727

Reynolds, S. P. 2003, in IAU Colloq. 192, Supernovae, (Berlin: Springer), in press (astro-ph/0308483)

Spitkovsky, A., \& Arons, J. 2004, ApJ, 603, 669 